\title{
An Integrated Robotic System: 2D-Vision based Inspection Robot with Automated PLC Conveyor System
}

\author{
Bandar Alghamdi, Dongbin Lee, Patrick Schaeffer, Joe Stuart \\ Oregon Institute of Technology \\ 3201 Campus Dr, Klamath Falls, USA \\ Bandar.Alghamdi@oit.edu; Don.Lee@ oit.edu; Pat.Schaeffer@ oit.edu; Joe.Stuart@oit.edu
}

\begin{abstract}
Automated quality control inspection is essential in manufacturing industries in these days. The purpose of this study is to improve product quality as well as increasing productivity by integrating three crucial systems in manufacturing lines; a robotic arm, 2D-vision, and conveyor system. As most of integrated systems have a tricky setup process to connect them to work as a single fully automated system, the process is a challenging, important step; for example, to connect a robot arm with 2D-vision based robotic inspection, and conveyor system controlled by a PLC, communicating with each other via communication network (Ethernet, Modbus). To determine main factors among selected characteristics, Minitab software is applied especially, to analyse 2D-vision system's ability and identify defective products while developing the entire process. A letter "O" was assigned as a testbed to simulate five defective work pieces: abrasion, chip, extra-material, pitting, and warp defect types, along with a template workpiece attached on Mock Bottle Cap. After two vision analyses using Minitab are applied to the integrated system, the result of 2D-vision inspection system is then able to identify four of the five simulated defects and the template workpiece, but not the warp defect due to the vision software with hardware. The vision inspection rate is over 95\% successful among the identified defects. Analysis of experimental data has been developed to find highly accurate relationship between conveyor motor speed (RPM) and the time interval to pick up defects by the robotic system for real-world applications with the help of Minitab software and tools of statistical analyses. Therefore, the research demonstrates that an integrated three-subsystem could have huge potential and promising in the future if we are able keep improving each subsystem while testing with a variety of different letters or shapes, to enhance manufacturing production line to increase quality control and quality assurance.
\end{abstract}

Keywords: Robot, Vision, Conveyor, Process Control, FANUC, PLC, Ethernet, Modbus, Integrated, Automation, Minitab, Statistical analysis, and Manufacturing Systems

\section{Introduction}

Automated systems have become an essential part of the manufacturing environment and production lines. Particularly an integration system, such as a robot with conveyor, conveyor with vision, robot with vision system, or mixed systems have seen significant growth in the last decades especially, in the auto industries or similar automated manufacturing lines. These systems are some of many choices in the field based on application and needs. Utilizing those integrated systems can bring a significant reduction in time consumption, human risk, and productivity cost. However, adding a new system or combining those key systems to work as a fully single automated system is a daunting challenge and is the essence of this study. The integrated system is considered as an inspection station in a factory line, in which it will identify defective products. Utilizing fully automated systems in industrial manufacturing will increase product quality, customer satisfaction, and allow more time for a company to train employees to adapt to the fast developing technology and advanced manufacturing facilities.

\section{Project Scope}

The first goal of this project is to combine the three systems to work as one fully integrated automated robotics and inspection system, utilizing a 6-axis FANUC LR-Mate 200iC robotic system, a 2D-vision system, and a conveyor system. The resulting system will be evaluated in terms of quality, accuracy, and precision. The project will help identify defects on a product that has been transferred by a conveyor belt and inspected by a 2D-vision system connected to a robotic arm, moving the product to the correct place based on the results of the inspection. Part of this process involves reviewing 
dimensions of individual products with the 2D-vision system. Applying supplementary inspection processes, such as a painting inspection with a 3D vision system, could be an option to identify another type of defect and improve the inspection process.

This project will identify the type of defects that can be determined, enhance the ability of the 2D-vision system to detect those defects, and provide benefits of using 2D-vision to the factory line. The project may come up with a solution to use a highly advanced vision system - such as a 3D-vision system, laser sensors, etc. - to yield better results in finding dimensional defects and painting defects. The improved efficiency of labour, use of energy, and cost of materials would increase productivity and enhance customer satisfaction through the consistent delivery of quality products. Figure 1 is the block diagram that has the components of the system used in this project except for PLC (see Figure 7 later).

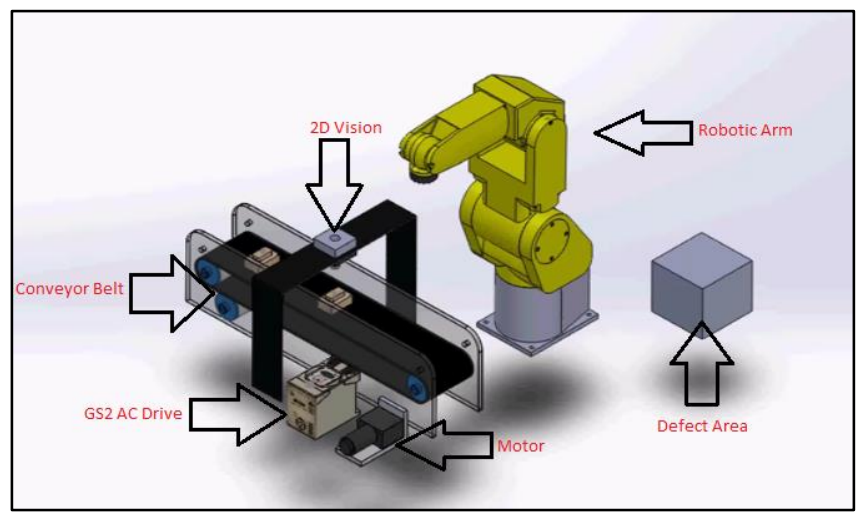

Fig. 1: Components of the proposed integrated system.

\section{Robotic System}

According to Fairhurst (1988), "The Robot Institute of America was reported in 1983 as defining a robot in the following terms: A robot is a programmable multifunction manipulator designed to move materials, parts, tools or specialized devices through variable programmed motions for the performance of variety of tasks" (p. 1).

In the past, human power was and still is the essential in any manufacturing industry. No matter how advanced and large the industry is, it needs an engineer to run, check, and fix any damages. Nowadays, almost all manufacturing companies use the certain type of technology, for example a robotic arm, to help them increase their productivity. There was a continuous increase in the market of industrial robots in the last decade. NASA states that it uses robotics for heavy and dangerous tasks that may put engineering experts at risk. The robotic arm is a machine that can do a specific job to increase accuracy and productivity, while reducing the time needed, the cost of unnecessary labour, and risk to humans (NASA 2009).

It is important to be aware of more popular robots around the globe. FANUC America has a variety of robots for different purposes. In this project, the LR Mate200iC with R-30iA controller series FANUC Robot Arm was used in this project. This version of the robot has six-axis joint for the 6-DOF motion of end-effector, i.e., three position and three angles. Refer to the FANUC user's manual for more information. In Figure 2, a 3D drawing shows the design for this particular robot; further specifications are available in the manual. Additionally, any robot company has a variety of robots beside on its size or application needed to be used for.

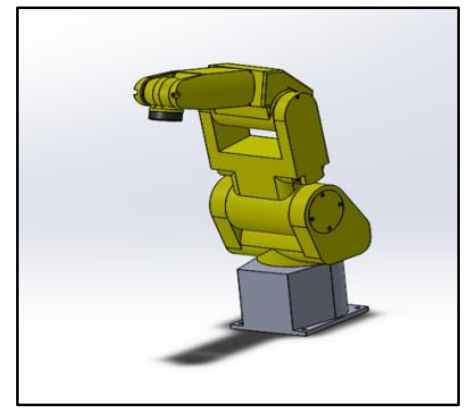

Fig. 2: 3D view of a Robot Arm. 


\section{2D-Vision System}

Vision inspection has a clear potential for beneficial applications, such as a visual system used in automation that quietly controls procedures in a manufacturing process. The inspection process could be used as a simple pass or fail decision to determine general appearance such as a number of items, shape, size, or defects for an object in order to control errors. Higher accuracy is the heart of an inspection process in an automation vision inspection (Fairhurst 1988. 9-10). A robot vision system is mounted on the top of a bridge fixed with conveyor system designed to take a quick inspection for sorting out the defective objects. When calibrating a robot to a vision system, essential factors need to be considered regardless of any vision inspection system used (Fairhurst 1988. 10-12). Without proper calibration or wrong setup of camera calibration, the quality of the robot's work suffers from and leads to inefficient projects and

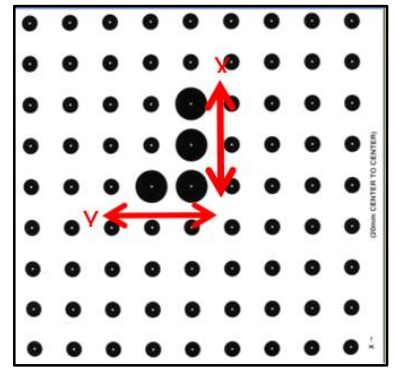

Fig. 3: 2D-Vision Calibration.

a potential loss of capital. Each robot has different calibration requirements based on their applications, such as different cameras dependent on the type of vision system used which accompanies the specific robot purchased. The difficulties in the process of developing vision control process to cope with were (1) Localization of object of interest, in which the camera must be calibrated to locate the workpiece under the exposure area shown in Figure 4, (2) Lighting conditions where the appropriate consistent lighting for the process must be determined prior to image collection, (3) Image segmentation in which appropriate time segments must be utilized for the camera to collect quality images, (4) Image quality as the black and white photography must be reviewed for high quality, and finally, processing speed as higher processing speeds increases the efficiency of the process. (Fairhurst 1988. 10-12).

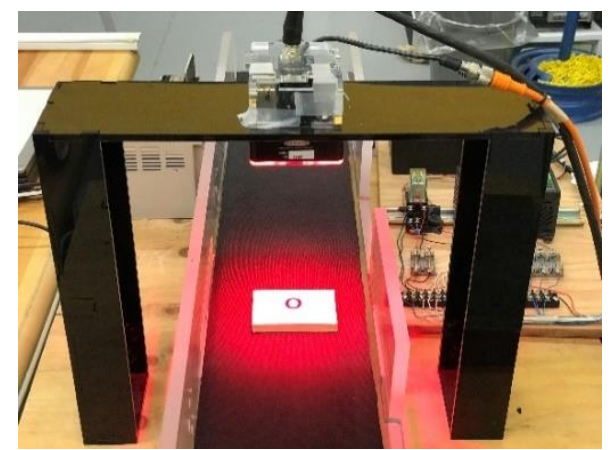

Fig. 4: 2D-Vision System w/LED Light.

In Figure 4, the robot used a specific camera with lens, B/W Progressive Scan Camera (Sony, 2017) and high-intensity LED ring light (Banner Engineering Corp) to work with the selected FANUC robot's vision software.

\section{Conveyor System}

\subsection{Design of Conveyor System}

All transportation machines, commonly referred to as conveyors, in every industry around the world have identical aspects in terms of the principle and the function of the conveyor. It is also considered as a small section of a larger application of material handling equipment; the main goal of using material handling machines is to move an object from one place to another. In other words, it doesn't increase the value of the parts, items, or pieces that are being moved. They 
don't shape, frame, process, or change an item in any way. Material handling applications involve raw materials, parts, boxes, crates, pallets, or luggage (McGuire 2010).

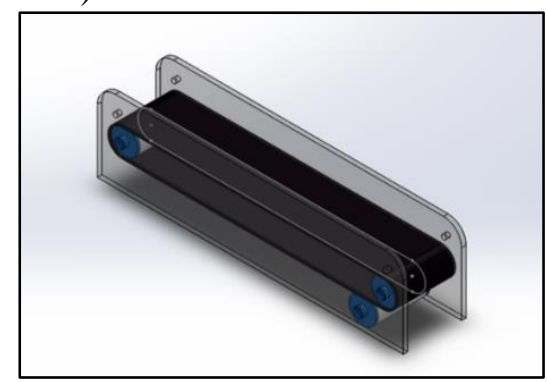

Fig. 5: 3D Drawing for the conveyor.

The Material Handling Institute of America offers the following as one definition of material handling: "Material handling is the art and science associated with providing the right materials to the right place in the right quantities, in the right condition, in the right sequence, in the right orientation, at the right time, at the right cost using the right methods" (McGuire 2010. 1). When companies consider the purpose of conveyors, such as how they can positively affect the overall outcome of the company, they are able to switch focus to further develop their productivity and product quality in the long run. This includes being aware of the cost of other operation that need more than one engineer, such as the installation process for any new machine purchased; e.g., Drive programming, mechanical and electrical installations, resource planning, operator training, periodic maintenance, and stocking spare parts on site (McGuire 2010).

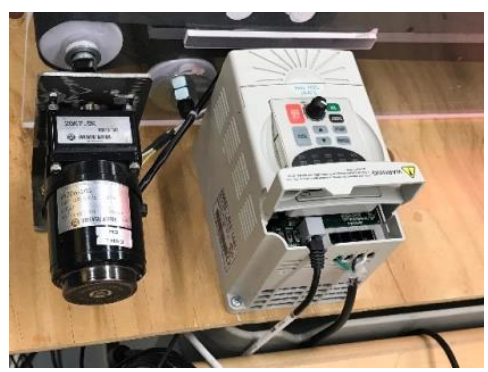

Fig. 6: An AC Motor with GS2.

\subsection{Motor Speed Control with AC Drive via PLC through Network}

The communication protocol is Modbus between programmable logic controller and AC motor drive (Automation Direct 2017) and Ethernet between the Robot and the PLC. In this project, an oriental motor (M206-202) with GS2 AC motor drive is controlled by user-defined PLC program in the DirectSoft 60 software as shown in Figure 6. For hardware calibration, several processes were setup for communication among the combined system, not only to install wiring configuration between the PLC and the rest of systems for application. In this project, a teaching wiring process has been used to highlight all components needed to building in preparation for programming. It is better to establish hardware configuration wiring as small as possible for ease of use and to connect to any system.

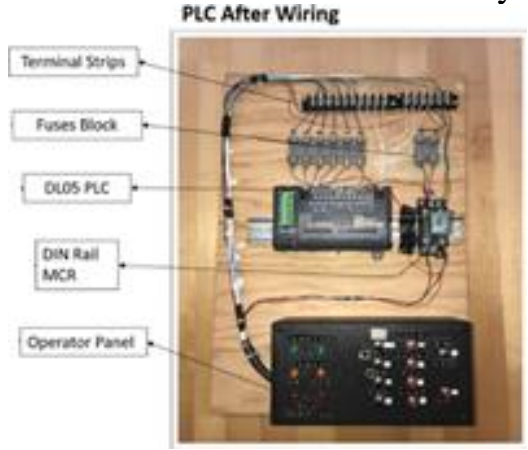

Fig. 7: PLC hardware configuration. 
Figure 7 shows the PLC before and after configuration wiring, and illustrate the main components used to build it.

\subsection{Total System Design: Distance, Speed, Pick-up}

After meeting all the requirements and options for the system, a ladder program can be constructed (“Automationdirect Technical Support - Example Programs" 2017). In the testing step, it is recommended to use an example provided by the company to troubleshoot the before starting the ladder program design. For instance, an example from Automation Direct called the First Run Program has been used to test the selected system ("GS2 Series Drives User Manual" 2017). The program has been created to work with multiple AC drives and PLC's. However, after testing the system and with few edits, the program for this project has been successfully created to work the application needed. There are two methods for the robot to pick up the defective product. The first method is picking up the product while the conveyer belt is moving, which requires two factors to be determined: the speed of the conveyor and the distance between each product placed on the conveyor surface. The equation below has been used to determine the appropriate speed. However, this is not exactly matched for this project for several reasons that will be discussed in problems, challenges, and solutions later.

$$
\begin{gathered}
\text { Angular Velocity }(\omega)=\frac{\text { Linear Velocity }(v)}{\text { Radius of Roll Diameter }(r)} \\
\text { Angular Velocity }(\omega)=2 \pi \div \text { Period }(T) \\
\text { Angular Velocity }(\omega)=2 \pi f(\text { frequency }) \\
R P M=\text { frequency }(f) \div 60
\end{gathered}
$$

With these formulas and algebra, we can find rpm:

$$
R P M=\frac{\text { Linear Velocity }(v) \times 4}{\text { Diameter }(d) \times \frac{30}{\pi} \text { seconds }}
$$

To calculate the distance between inspection point and robot pick-up point as well as the conveyor velocity to feed the object. With given above formula, below graph is drawn based on the ideal RPM (speed) of the conveyor on the X-axis and the time interval on the $y$-axis; if conveyor increases the rpm, robot can reduce the pick-up time. Ideal RPM is calculated how fast linearly mocking bottle cap will move due to the rpm on the motor based on the size of conveyor. With these data, the appropriate distance is obtained how far the bottle cap moves from the inspection point to the robot pick-up point. For example, through the project we determined the distance which is 11.5 [in]. As a result, it was possible to know what inputs of the PLC must be used to move the block from the scanning area to where the robot picks up the mock cap.

Table 1: Robot Time to pick-up vs. Conveyor RPM: roller diameter $=6$ in, pick-up distance $=11.5 \mathrm{in}$.

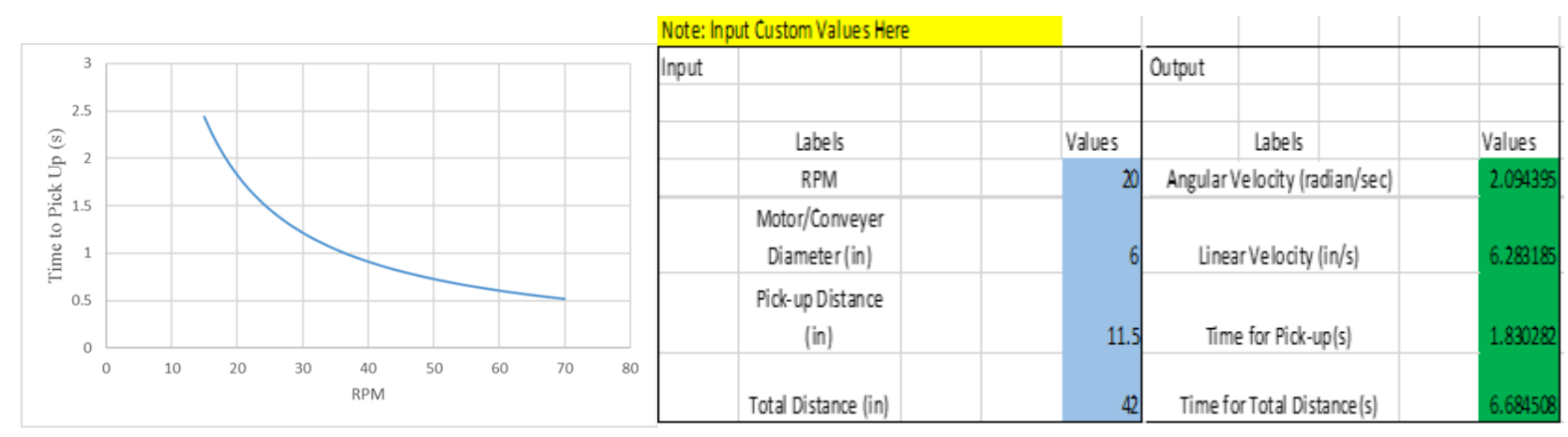


The second method that can be used in this project is picking up the defect product while the conveyor belt is stopped. Here the conveyor need to be programmed by the PLC to start and stop in a current time, which will allow the robot to pick up the defective product while the other product being inspect.

\section{Data and Analysis}

It is important to evaluate every new system to identify its functions and how it can make different during inspection process. Therefore, two analysis tests have been done in this project to prove the efficiency of the 2D-vision software and to determine what tools should be used in the inspection process. All the data and images are available for more information. The analysis has been done by (Minitab 17). The five-workpiece images with the template for the test are shown below Figure 8.

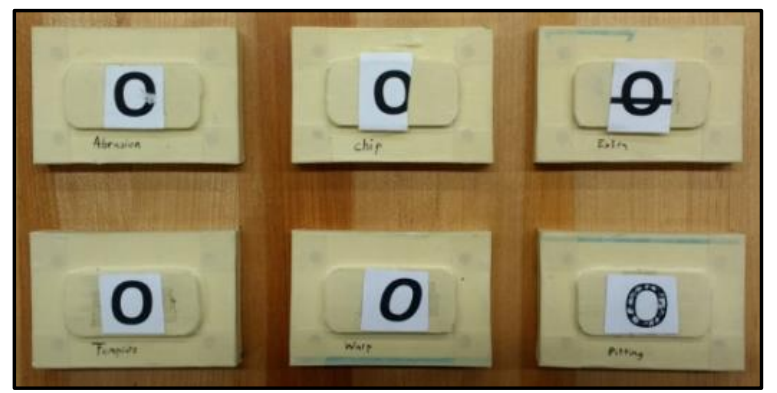

Fig. 8: PLC hardware configuration.

\subsection{First Analysis}

In this analysis, all factors have been used and coded (tools, position, angels, type of defects) to identify which is significant to use during the inspection process as shows in Figure 8 below.
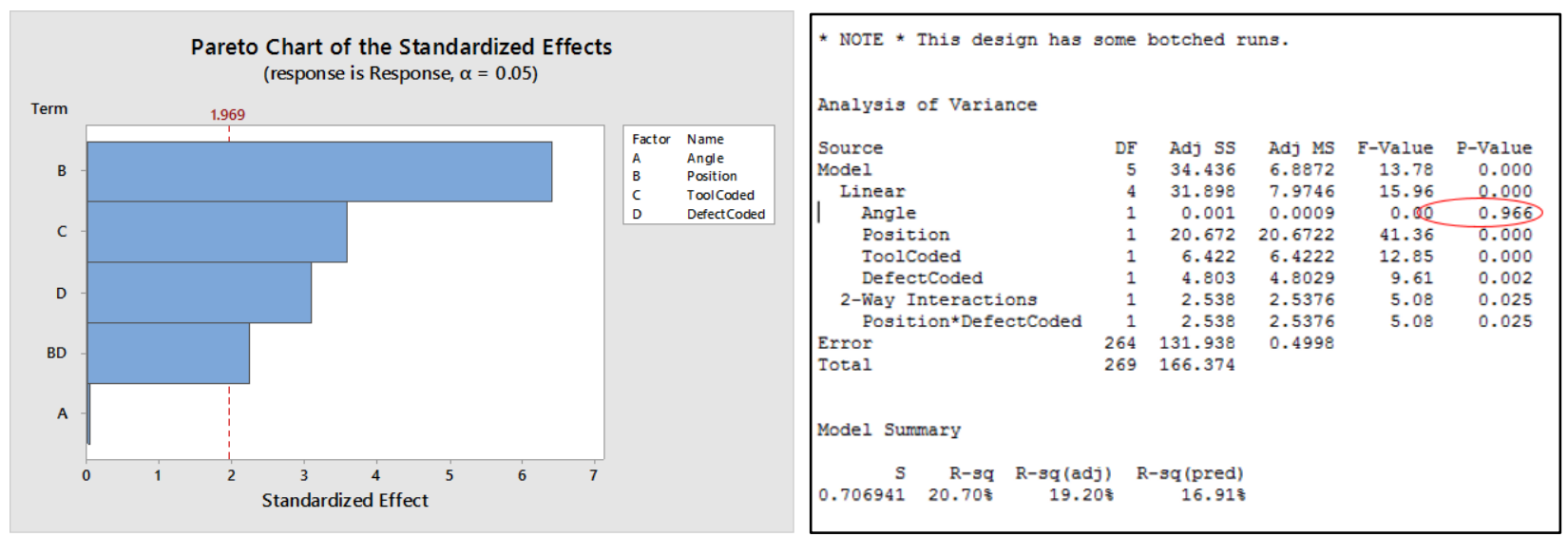

Fig. 9: Factorial Regression Response Vs Angle, Position, Tool-Coded, and Defect-coded after reduction.

Figure 9 shows the reduced model using the mean response is listed above, which illustrate that angles, positions, tool-coded, and defect-coded was therefore reduced out of the model due to the factor interaction effect was 'small' (an order of magnitude) compared to the main effects. Therefore, the factors positions, tool-coded, and defect-coded along with the interaction position*defect-coded are SIGNIFCANT. It is also show that Angle is NOT significant after the reduction $=0.966$, RULE: If $\mathrm{p}<0.05$, then the factor is significant. 


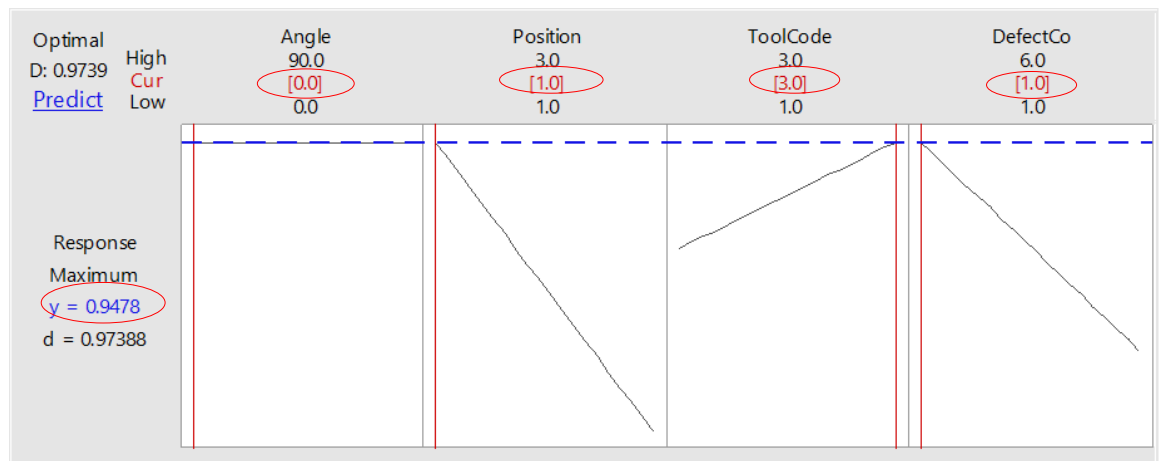

Fig. 10: optimization plot shows detectability score: 0.95, angel 0, position 1, tool 3 (histogram), and defect 1 (abrasion).

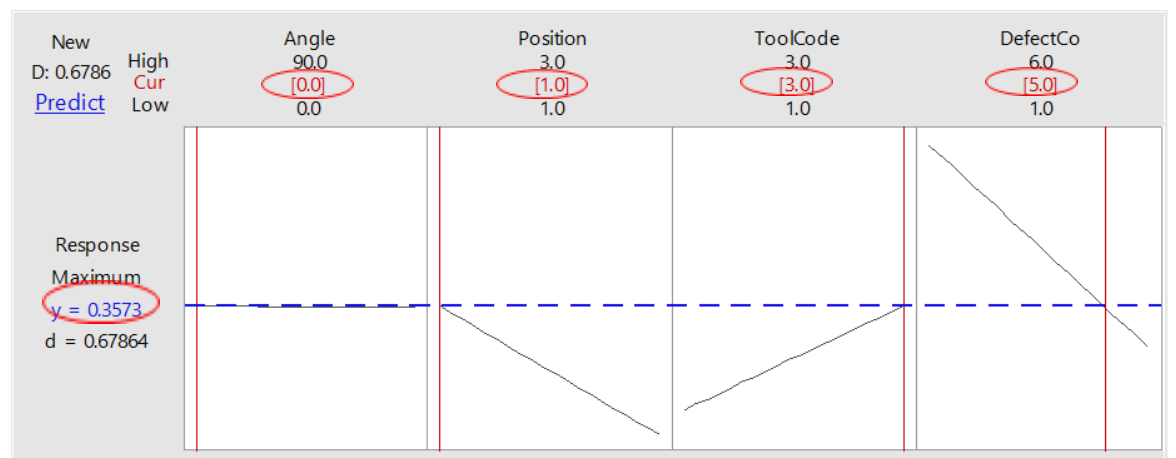

Fig. 11: optimization plot shows the detectability score: 0.357 , angel 0 , position 1 , tool 3(histogram), and defect 5 (template).

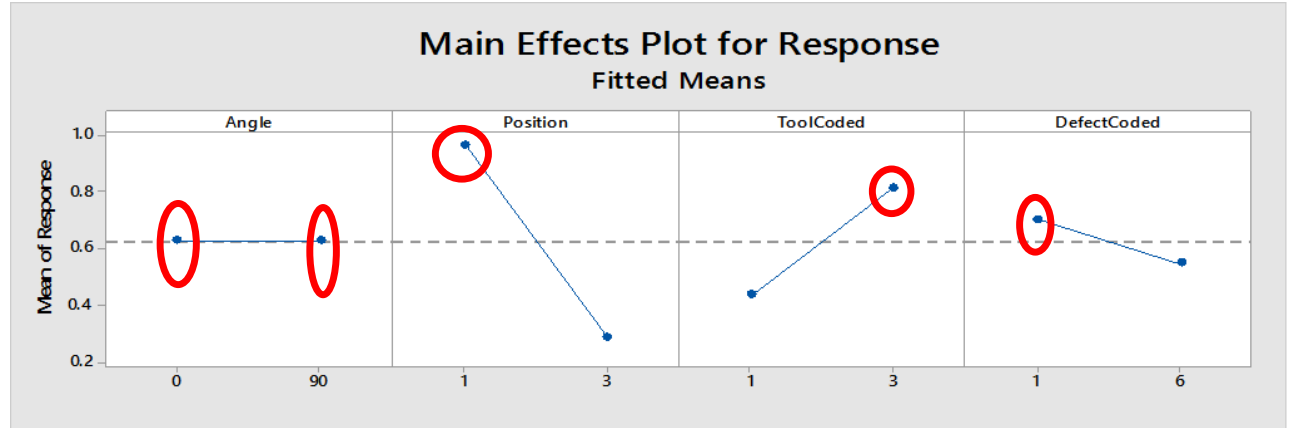

Fig. 12: main effects plot response with equal angle 0-90, position 1, tool 3(histogram), and defect 1 (abrasion).

In Figures 12, angle factor is NOT significant in this process, and also show the highest point of position 1, toll-coded 3 (histogram tool), and unfortunately, heist defect-coded 1 (abrasion); thus, this analysis has filed to prove the efficacy of this process, in which Figure 10 illustrates the highest detectability score of 0.95; however, Figure 11 shows the abrasion defect type while the template part has detectability score of 0.357 only as a result of that, the next analysis will try to prove the efficacy of the process after eliminating not significant factor like angles.

\subsection{Second Analysis}

In this analysis all factors (tools, position, and type of defects) have been used and coded to identify which is significant to use during the inspection process as shows in the tables below. 

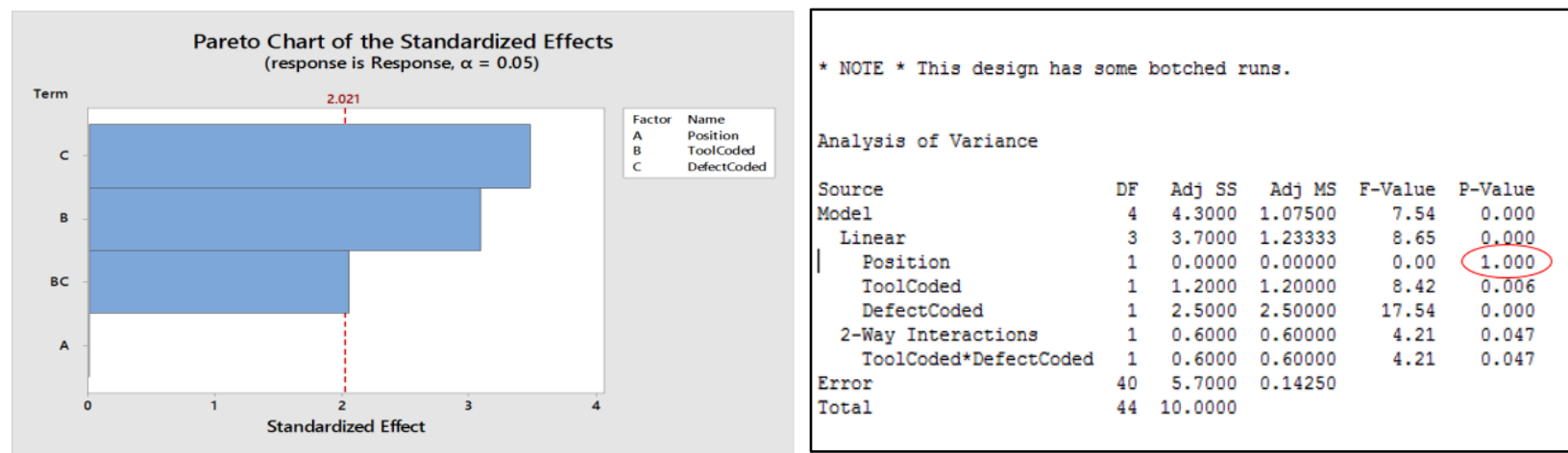

Fig. 13: All significant factors along with the interaction (tool-coded*defect-coded) except position= 1.000 .

Figure 13 shows the average at the high level - the average at the low level of the standard privation, which is 2.021, and factor A (position) is NOT significant and the factors B (tool-coded), C (defect-coded), and BC (tool-coded *defect-coded) are significant factors.

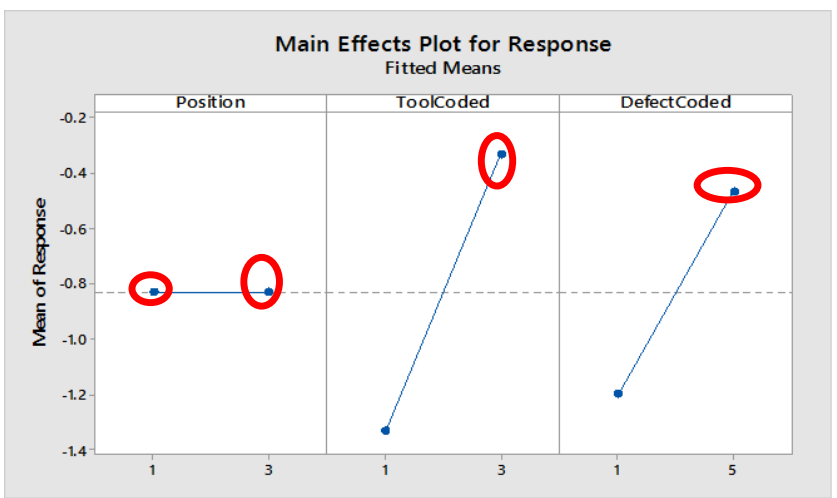

Fig. 14: Plot of Main Effects for Response with equal position 1-3, tool 3(histogram), and defect 5 (template).

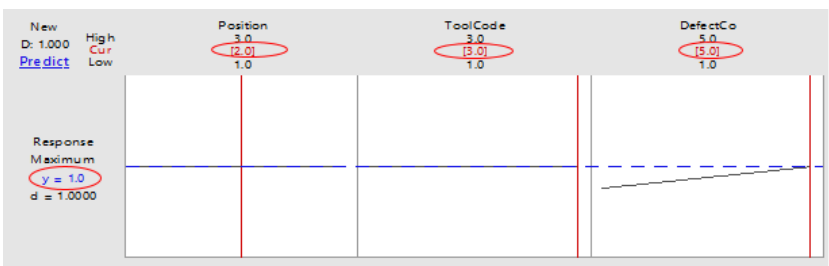

Fig. 15: Detectability Score of 1.0 on defect 5 (template), with tool 3(histogram tool) and position 2.

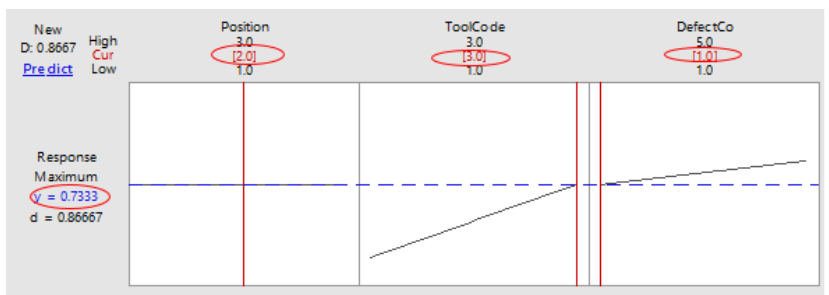

Fig. 16: Detectability Score of 0.733 on defect 1 (abrasion), with tool 1(histogram tool) and position 2.

In Figures 14, the position factor is not significant while showing the highest point of tool 3 (histogram) and the highest point of the defect 5 (template). The plots show the main effects, in which the position is NOT significant in this process. Figure 15 and 16 show that detectability score is 1.0/.733 on defect 5/1 (template/abrasion) with the point 
of tool 3/1 (histogram) and position 2, respectively. Thus, the template workpiece is able to be located by any tool in the 2D-vision software at any position.

\section{Conclusion}

Most of the large manufacturing industries utilize automated two systems, such as robotic with vison, conveyor with vision, or robot system with conveyor. Utilizing more systems to work as one integrated system may be the solution to reduce defective products, time-consuming product manufacturing, and labour cost. Applying quality control integration systems will create quality product, increasing productivity that results in company's long-term growth and customer satisfaction.

An integrated system, consisting of a robotic system, vision w/light, and automated conveyor are implemented and tested to get reasonable results that are shown in the Figures and Tables. During developing the automated process, vision identification is more important than the others. The contribution of the system in the paper can be above all improved accuracy and efficiency through an analysis tool. In order to get quality identification, two vision analyses using Minitab are applied; the first analysis is to find major factors to identify significant factors which determines quality identification. Among five factors selected, position of the letter on the cap and angle of shape were dropped due to the fact the two factors don't affect the quality, which means the vision system detect well no matter where they are located or no matter how much it was rotated. In the second analysis five defectives such as abrasion, chip, extra-material, pit, and warp with the template are used to analyse the performance of the vision system using Minitab analysis and obtained over 95\% detection of four of five factors (work pieces) but we also found a weakness that warp model was not able to identify well due to the limitation of SW/HW version but the affined model detection problem should work in the future and potentially would overcome with updated vision algorithms and/or hardware such as 3D laser with vision system. Secondly, the rpmtime curve is obtained from equations and further developed to estimate with less sum-of-square-errors by curve-fitting for finding the exact time to pick up material using the robotic arm from start-stop conveyor motion. Thirdly, all factors are considered for the conveyor system such as the type of the application, product specifications, the size of the conveyor and so on, which are very important factors to increase the speed and accuracy of the inspection process. The conveyor system is controlled by PLC via Modbus communication protocol and material handling by the robot is communicated with Ethernet communication between PLC and the robot controller. Finally, a single integrated whole subsystem such as vision system, PLC, and conveyor are built and tested and then, the results are shown in the Figures with Tables. This study verifies that the proposed system can be a good reference for existing automated systems and prove the effectiveness of the combined integrated systems.

\section{Acknowledgements}

The authors would like to thank Dr. Jeffrey Hayen's support as MMET Chairperson, enabling us to utilize facilities and administrative work at Oregon Tech. Special thanks to Mr. Minar who helped us to establish the system. We also sincerely appreciate to the project sponsor, Saudi Arabian Culture mission (SACM), which supported the project financially and especially, for Bandar for his research throughout the graduate degree.

\section{References}

[1] C. Michael Fairhurst, Computer VISION FOR ROBOTIC SYSTEMS, Hemel Hempsteadz, Prentice Hall International (UK) Ltd., 1988.

[2] W. Kölbl, "Affordable Optical Seam Tracking- Meta Torch Systems Break the Price Barrier," Industrial Robot, vol. 22, no. 6, pp. 19-21, 1995. doi: http://dx.doi.org/10.1108/01439919510105110

[3] Banner Engineering Corp, LEBRR70X\&0-78587 Ring Light. Banner Engineering Corp., Nov. 2012. [Online]. Available: http://info.bannerengineering.com/cs/groups/public/documents/literature/134088.pdf

[4] A. Hand, "ABB to Acquire Factory Automation Player B\&R," Automation World, 2017. [Online]. Available: https://www.automationworld.com/plcs-pacs/abb-acquire-factory-automation-player-br

[5] R. Lucas, "Systems Integration," ICL Technical Journal, vol. 6 no. 3, pp. 415-534, 1989.

[6] P. M. McGuire, Conveyors: Application, Selection, and Integration. Boca Raton, FL: CRC Press, 2009

[7] P. Novak, "The Basics of Variable-Frequency Drives," Electrical Construction and Maintenance, 2009, Fluor, Inc. [Online]. Available: http://ecmweb.com/power-quality/basics-variable-frequency-drives 
[8] B. Rooks, "A history of reliability and service for ABB's robots at clares," Industrial Robot: An International Journal, vol. 22, no. 6, p. 33, 1995. doi:10.1108/01439919510105165.

[9] J. Testa, "The vision-guided robot grows up - a look at the new machine vision technology for robots," Industrial Robot: An International Journal, vol. 22, no. 6, pp. 13-15, 1995.

[10] V. Volcovic, "Apple's robot rips apart iPhones for recycling," Thomson Reuters, 2016. [Online]. Available: http://www.reuters.com/article/us-apple-products-recycling-idUSKCNOWN1Y0

[11] E.St. Wienand, "Vision system for robot guidance and quality measurement systems in automotive industry," Industrial Robot: An Intl Journal, vol. 22, no. 6, pp. 26-29. doi: http://dx.doi.org/10.1108/0143991951010514.

[12] Automationdirect.com, "GS2 Series Drives User Manual \& EP-COM-003: Networking Multiple GS Drives with the Standard Instruction Set in the DL05 and D2-250 \& DL05 User Manual," [Online]. Available: http://support.automationdirect.com/examples.html\#ct and so on.

[13] FANUC Robotics America, Inc. 2007. iRVision Operator's Manual \& 2D Vision START-UP GUIDANCE. Rochester Hills, MI: FANUC Robotics America, Inc. [Online]. Available: http://robot.fanucamerica.com/products/vision-software/robot-vision-software.aspx 